\title{
Congenital Colonic Atresia: an infrequent cause of low intestinal obstruction in neonates
}

\author{
Authors \\ Manas Shanbhag ${ }^{1}$, Mrudula Pala ${ }^{2}$, Murali Keshava S. $^{3}$ \\ ${ }^{1}$ Assistant Professor, ${ }^{2}$ Junior Resident, ${ }^{3}$ Assistant Professor \\ Department of Paediatrics, Kasturba Medical College, Mangaluru, \\ Manipal Academy of Higher Education \\ Corresponding Author \\ Dr Manas Shanbhag \\ Department of Paediatrics, Kasturba Medical College, Mangaluru -575001 \\ Manipal Academy of Higher Education \\ Mobile: +91 9886047617, Email: manas.shanbhag@gmail.com
}

\begin{abstract}
Colonic atresia is a rare cause of distal intestinal obstruction in neonates. In this report a two day old neonate who presented with abdominal distension, bilious vomiting and failure to pass meconium due to congenital colonic atresia is being described for its rarity. Congenital colonic atresia should be considered in the differential diagnosis when a neonate presents with features suggestive of intestinal obstruction.
\end{abstract}

Keywords: Colonic atresia, Intestinal obstruction, Failure to pass meconium, Abdominal distension, Bilious vomiting.

\section{Introduction}

Colon atresia comprises 1.8 to 15 percent of all GI atresia and incidence varies from 1:5000 to 1:60,000. First case of colon atresia was reported in 1673 by Binninger. Gaub reported the first survivor in 1922, an infant with sigmoid colon atresia who was treated with delayed colo-colic anastomosis after primary colostomy. Isolated colon atresia is frequently associated with skeletal anomalies such as syndactyly, polydactyly, absent radius and club foot. They may be complicated by abdominal wall defects like gastroschisis, omphalocele, and vesicointestinal fistula.

\section{Case report}

A female baby weighing $2.5 \mathrm{kgs}$ was delivered after full term pregnancy. Two days after birth she got admitted with complaints of abdominal distension, bilious vomiting and failure to pass meconium. Physical examination showed a distended abdomen with no associated anomalies. Abdominal x-ray showed multiple air fluid levels with no air in rectum. Urograffin enema studies showed microcolon with proximal bowel loops distension. Surgical exploration showed grossly dilated colon with blind end at junction of descending to sigmoid colon with distal loop microcolon. Primary colostomy done, distal colon biopsy sent for HPE 
showed presence of neuroganglion cells with atretic colon segment. Baby recovered clinically and discharged on post natal day 11 with colostomyinsitu with a plan to do pull through surgery after a year.

\section{Discussion}

Colon atresia is one of the rarest intestinal obstruction. Several theories are there to explain the aetiology of colon atresia. The main one was intra uterine extrinsic mesenteric vascular obstruction which can be caused by internal hernia, volvulus, intussusception or a strangulation in tight gastroschisis $^{1,2}$. Intraluminal vascular factors have also been reported. Erskine proposed emboli originating from placenta as anaetiology for colon atresia $^{3}$. Foetal infection with varicella can cause injury to enteric plexus leading to poor vessel development and induce ischemic conditions resulting in colon atresia ${ }^{4}$. Familial causes have been reported ${ }^{5}$. Newborns with colon atresia presents with distal bowel obstruction. Plain radiograph of abdomen reveals dilated bowel loops with air fluid levels, contrast enema will identify the narrowed colon distal to obstruction. Barium can be dangerous if a perforation occurs during examination hence non-ionic soluble contrast material is preferred. If the entire sigmoid and upper rectum are involved in atresia colostomy followed by Swenson pull through operation is best done at one year of age, this procedure provides excellent postoperative colon function ${ }^{6}$. When a colon atresia has been repaired successfully but patient remains partially obstructed an unsuspected small bowel stenosis or upper segment dilatation should be sought by upper GI contrast studies. Thorough exploration of entire intestinal tract and rectal biopsy at the first operation seems to be an important step because Hirschsprung's disease may complicate colon atresia. Unlike proximal bowel atresia, distal atresia diagnosis was commonly delayed until feeding have been started, this delay leads to more severe dehydration, sepsis, higher mortality rates ${ }^{7}$. Significant high mortality rates in cases operated after $72 \mathrm{hrs}$ seems to underlie the importance of early diagnosis and treatment.

\section{References}

1. Potts WJ (1947) Congenital atresia of intestine and colon. Surg Gynec Obstet 85(1):14-19.

2. Benson CD, Lofti MW, BroghAJ (1968) Congenital atresia and stenosis of the colon $\mathrm{J}$ Pediatr Surg 3(2):253-257.

3. Erskine JM (1970) colonic stenosis in the newborn: the possible thromboembolic etiology of intestinal stenosis and atresia. J Pediatr Surg 5(3):321-333.

4. Alexander I (1979) Congenital varicella.Br Med J 2(6197):1074.

5. Guttman FM, Braun $\mathrm{P}$, Garance $\mathrm{PH}$, Blanchard H, Collin PP, et al.(1973) Multiple atresias and a new syndrome of hereditary multiple atresias involving the GIT from stomach to rectum .J Pediatric Surg8(5):633-640.

6. Powell RW, Raffensperger JG (1982) Congenital colonic atresia. J Pediatr Surg 17(2):166-170.

7. Karnak I, Ciftci AO, Senocak ME, Tanyel FC, Buyukpamukcu N(2001) Colonic atresia: surgical management and outcome. Pediatr Surg Int 17(8):631-635. 